\title{
Understanding the Activity of Antibiotics in Cerebrospinal Fluid in vitro
}

\author{
Peter Matzneller ${ }^{a}$ Angela Burian ${ }^{a, b}$ Markus Zeitlinger ${ }^{a}$ Robert Sauermann ${ }^{a, c}$ \\ a Department of Clinical Pharmacology and bepartment of Physical Medicine and Rehabilitation, Medical \\ University of Vienna, Vienna, and ' Main Association of the Austrian Social Security Institutions, Vienna, Austria
}

\section{Key Words}

Antibiotics · Cerebrospinal fluid $\cdot$ Infection $\cdot$ In vitro
Methodological recommendations derived from the collected studies are summarized in order to optimize future research on the topic.

(c) 2016 S. Karger AG, Basel

\begin{abstract}
Background: In vitro studies suggest that antimicrobial activity of antibiotics meant to treat central nervous system infections such as meningitis or ventriculitis may be altered by cerebrospinal fluid (CSF). This could explain the reason behind the often observed discrepancies between the activity of antibiotics determined in artificial growth media in vitro, and their sometimes reduced clinical efficacy in CSF in vivo. If conducted in CSF, in vitro microbiological investigations might predict the ability of antibiotic drugs to treat CSF infections better than experiments in artificial growth media. In addition, they are less expensive, critical and time consuming than animal studies, and might potentially be appreciated in drug development as a rapid and cost-effective means to gain valuable information on drugs meant to treat infections residing in CSF. Summary: Data from microbiological in vitro experiments performed in CSF were compiled for fosfomycin, rifampicin, cefepime, cefotaxime, ceftriaxone, ciprofloxacin, gentamicin and vancomycin. Where possible, correlations between in vitro data and evidence from in vivo studies were established. Key Messages: As discussed in the text, no clear correlations between in vitro studies in CSF and clinical outcomes could be identified.
\end{abstract}

\section{Introduction}

Despite the broad range of available antibiotics, bacterial infections residing in cerebrospinal fluid (CSF) like meningitis or ventriculitis remain challenging for clinicians due to their clinical severity. Because sufficient antibiotic concentrations at the site of infection are essential for the success of antimicrobial therapy, many studies have focused on determining antibiotic penetration into CSF. In contrast, only a few efforts have been directed to studying the influence of CSF per se on pharmacodynamics, that is, on antimicrobial activity. However, a limited number of microbiological in vitro experiments indicate that the activity of some antibiotics may be altered in CSF, which could affect their efficacy in the treatment of central nervous system (CNS) infections in vivo.

The potential influence of CSF on antibiotic activity could explain why many clinical trials investigating antimicrobial therapy of CNS infections conclude that the minimum inhibitory concentration (MIC) of the tested agent should be exceeded by several fold in CSF of patients with

\section{KARGER}

E-Mail karger@karger.com

www.karger.com/pha
(C) 2016 S. Karger AG, Basel

0031-7012/16/0976-0233\$39.50/0
Markus Zeitlinger

Department of Clinical Pharmacology

Medical University of Vienna

Währinger Gürtel 18-20, AT-1090 Vienna (Austria)

E-Mail markus.zeitlinger@meduniwien.ac.at 
meningitis [1-3]. Accordingly, apparent discrepancies between in vitro results and in vivo efficacy or the divergence between theoretically 'inhibitory' drug concentrations in CSF in vivo and poor clinical outcomes [4,5], might be attributed to the fact that bacterial susceptibility tests are conducted mainly in artificial media and not in CSF.

Experimental meningitis models in animals are a good method for evaluating the activity of antibiotics in CSF. However, they are relatively expensive, laborious and should be avoided when not necessary. So the question arises if it is meaningful to test the activity of antimicrobial agents in CSF in vitro to estimate if they would be eligible for treatment of CNS infections and to minimize the number of required animal studies due to optimal pre-selection of antibiotics. Microbiological in vitro experiments in CSF are relatively cheap, easy to perform and less time-consuming than animal studies, and might therefore be appealing for researchers involved in development of antimicrobial drugs meant to treat infections residing in CSF. However, selection of antibiotics for further evaluation in CNS infection models based on in vitro studies would be meaningful only if there is a good correlation of such in vitro results with in vivo activity in CSF.

Therefore, the central focus of this review is to compile and interpret data on antibiotic activity in CSF in vitro vs. in vivo, whereas other aspects were regarded with less intensity. A search of the literature was performed to identify studies performing microbiological experiments using CSF in vitro. Antibiotics are described here only if data of (a) in vitro experiments in CSF (and other media), and (b) in vivo studies involving treatment of CNS infections as monotherapy, preferably reporting bacterial counts in CSF, were available and could be compared.

To our knowledge, this review article constitutes the first attempt to describe the ability of microbiological in vitro experiments in CSF to predict the clinical efficacy of antimicrobial drugs meant to treat severe CNS infections like meningitis or ventriculitis. Limitations of the studies compiled here and methodological recommendations derived from them are summarized at the end of this article, with the intent to improve future research on the topic.

\section{CSF}

\section{Properties of Normal CSF}

CSF is an ultrafiltrate of plasma arising from the choroid plexus in the ventricles at a rate of $\sim 0.5 \mathrm{ml} / \mathrm{min}$ in adults. It is also contained in the subarachnoid space
Table 1. Characteristics of healthy CSF $[8,9]$

\begin{tabular}{|c|c|}
\hline Compound & Concentration \\
\hline Protein (lumbar CSF), g/l & $0.1-0.4$ \\
\hline Protein (ventricular CSF), g/l & $0.07-0.25$ \\
\hline Glucose, $\mathrm{mmol} / \mathrm{l}$ & $2.8-4.4$ \\
\hline Sodium, mmol/l & $135-150$ \\
\hline Potassium, mmol/l & $2.6-3.0$ \\
\hline Chloride, $\mathrm{mmol} / \mathrm{l}$ & $115-130$ \\
\hline Calcium, mmol/l & $1.00-1.40$ \\
\hline Magnesium, mmol/l & $1.2-1.5$ \\
\hline Phosphorus, mmol/l & $0.4-0.6$ \\
\hline Iron, $\mathrm{mmol} / \mathrm{l}$ & $0.2-0.4$ \\
\hline Creatinine, mmol/l & $50-110$ \\
\hline Urea, mmol/l & $3.0-6.5$ \\
\hline Lactate, $\mathrm{mmol} / \mathrm{l}$ & $1.1-2.4$ \\
\hline Carbon dioxide, mmol/l & $20-25$ \\
\hline $\mathrm{PCO}_{2}, \mathrm{~mm} \mathrm{Hg}$ & $44-50$ \\
\hline $\mathrm{PO}_{2}, \mathrm{~mm} \mathrm{Hg}$ & $40-44$ \\
\hline $\mathrm{pH}$ & $7.28-7.32$ \\
\hline Osmolality, mOsm/ $\mathrm{kg} \mathrm{H}_{2} \mathrm{O}$ & $280-300$ \\
\hline Cells & $<5 / \mathrm{mm}^{3}$, no erythrocytes \\
\hline
\end{tabular}

bathing the brain and the spinal cord. The total volume of CSF is $\sim 150 \mathrm{ml}$. Finally, CSF is drained through the subarachnoid granulations into the superior sagittal sinus and also through the brain extracellular space. Overall, CSF is exchanged approximately 4 times per day [6].

The most important physiological functions of CSF are transportation of neuroendocrine factors, elimination of metabolic waste products and mechanical protection of the brain from injury by acting as cushion [7].

The normal composition of human CSF is shown in table $1[8,9]$. Most constituents of CSF have equal or lower concentrations than in plasma. Almost all proteins in CSF are derived from serum with the exception of some trace proteins originating in the brain. CSF normally contains a small number of lymphocytes and monocytes with a total cell count of $<5$ cells $/ \mathrm{mm}^{3}$.

\section{Pathologic Alterations of CSF}

Pathologic processes of the CNS are characterized by alterations in the composition of CSF. In experimental meningitis, the production of CSF is reduced [10]. Moreover, meningitis increases the outflow resistance of CSF, leading to a decreased CSF bulk flow. A decrease in CSF bulk flow can increase the half-life of drugs in CSF. Also, an adjunctive therapy with corticosteroids inhibits the secretion of CSF by approximately $30 \%$ in bacterial meningitis [6]. However, antibiotic concentrations often appear to be unaltered by concomitant steroid administration [6]. 
An increase in cell counts indicates an infection of the CNS, or other pathological CNS conditions. The differential cell count provides further information regarding the possible cause of the CNS disease. During bacterial meningitis, the protein content of lumbar CSF rises usually to $\sim 5-10$ times the normal value due to an increased permeability of the blood-brain barrier. In chronic infections like tuberculous meningitis, the protein value can reach even higher levels. In viral CNS infections, the protein content of the CSF often amounts to 2 or 3 times the normal value [11]. The CSF glucose levels are essentially normal in viral infections, but maybe significantly lowered in bacterial meningitis.

\section{CSF as Microbiological Medium}

Because a number of bacterial infections may reside in CSF, and because CSF has a characteristic composition, CSF has been used as in vitro medium for microbiologic experiments by several researchers to study the effects of antimicrobial agents. However, a problem of in vitro studies with CSF is that the $\mathrm{pH}$ of CSF increases significantly after sampling from patients or animals. Significant changes in $\mathrm{pH}$ after sampling of CSF may affect survival, growth and antibiotic killing of bacterial strains in vitro $[12,13]$. When the $\mathrm{pH}$ of CSF was tested in vitro under different environmental conditions (in ambient air at $4^{\circ} \mathrm{C}$, in ambient air at $22^{\circ} \mathrm{C}$, in ambient air at $37^{\circ} \mathrm{C}$, and in air with $5 \% \mathrm{CO}_{2}$ at $37^{\circ} \mathrm{C}$ ), the $\mathrm{pH}$ of $\mathrm{CSF}$ significantly increased under all tested conditions with the exception of air with $5 \% \mathrm{CO}_{2}$ at $37^{\circ} \mathrm{C}$ where it remained stable [12]. Apparently, this increase in $\mathrm{pH}$ occurs due the loss of $\mathrm{CO}_{2}$ from CSF to ambient air; this can be avoided by the incubation of CSF in an atmosphere containing $\mathrm{CO}_{2}$. Also the best survival of different bacterial species was observed in CSF incubated in air with 5\% $\mathrm{CO}_{2}$ at $37^{\circ} \mathrm{C}$ [12]. Thus, in vitro microbiologic experiments may produce misleading results if they are performed in CSF with increased $\mathrm{pH}$. Therefore, in this review, special attention is paid to the description of $\mathrm{pH}$ adjustment in the cited articles.

Another important observation regarding CSF as microbiologic medium is that bacterial growth tends to be generally lower than in other liquid standard media, like Mueller Hinton broth (MHB). Good bacterial growth in professional microbiologic media can be attributed to the higher content of nutrients such as glucose or select electrolytes compared to CSF. Importantly, such altered growth rates are expected to affect bacterial killing rates of antibiotics, in particular, of drugs which act 'growthdependently'.

Antibiotics in CSF

\section{Antimicrobial Activity of Antibiotics in CSF}

It is the aim of this section to report the essential findings from the literature on the activity of several antibiotics in CSF in vitro and in vivo. The activity of the individual antibiotics in CSF in vitro is compared with the respective in vivo findings observed either in animals or in humans with experimental or clinical CSF infections like meningitis or ventriculitis, respectively. Therefore, microbiological in vitro tests are considered and reported here only when in vivo data are also available for the same drug used to treat CSF infections. In addition, accompanying in vitro tests in standard media (broth) are described in the publications, if they were used for direct comparison with activity in CSF in vivo.

For this purpose, a search of the literature in English, French, German and Spanish language was performed using the key words cerebrospinal fluid, liquor, time-kill curve, killing, bacterial killing, antibiotic, antimicrobial activity, growth curve, growth inhibition, MIC, in vitro in multiple combinations. If microbiological in vitro experiments in CSF were found, it was checked if there are also in vivo data available indicating the activity of these antimicrobial drugs when used for treatment of CSF infections. It was beyond the scope of this review to report all available studies using experimental meningitis models.

\section{Fosfomycin}

Mechanism of Action

Fosfomycin irreversibly inactivates the UDP-N-acetylglucosamine enolpyruvyl transferase, leading to the discontinuation of murein synthesis in the bacterial cell wall. Its bactericidal activity was reported to be time-dependent; hence, its activity is optimized if the MIC is exceeded as long as possible at the site of infection [14]. After intravenous administration, fosfomycin has been shown to reach CSF concentrations greatly exceeding the MIC of a wide range of clinically relevant pathogens, although plasma-CSF equilibration remains incomplete [14-17].

\section{In vitro}

Time-kill curves were performed in MHB and in CSF in vitro using one clinical Staphylococcus aureus strain and static fosfomycin concentrations ranging from 0.25 to 8 times the MIC to evaluate the influence of CSF on the activity of fosfomycin [18]. MHB was supplemented with glucose-6-phosphate (G-6-P) as required for susceptibility tests. Pooled human CSF was incubated both in ambient air and in air with $5 \% \mathrm{CO}_{2}$ for adjustment of $\mathrm{pH}$ to physiological values. 
Time-kill curves in CSF in ambient air showed high $\mathrm{pH}$ of CSF and almost no activity of fosfomycin even at 8 times the MIC, confirming the importance of using $\mathrm{pH}$ adjusted media. Time-kill curves in $\mathrm{pH}$-adjusted CSF showed initial killing between 0 and $8 \mathrm{~h}$ at fosfomycin concentrations equal to or higher than the MIC $(\geq 1 \times$ MIC). Velocity of killing from 0 to $8 \mathrm{~h}$ was similar in CSF and MHB. However, in CSF, bacterial regrowth was observed between 8 and $24 \mathrm{~h}$ at all concentrations $\leq 4 \times$ MIC, and sustained killing at $24 \mathrm{~h}$ occurred only at 8 times the MIC. In contrast, in MHB sustained killing was achieved at concentrations $\geq 1 \times$ MIC.

\section{In vivo}

Ribes et al. [19] tested fosfomycin monotherapy to treat experimental meningitis in rabbits induced by 2 penicillin- and cephalosporin-resistant strains of Streptococcus pneumoniae. MICs of the 2 test strains were 4 and $16 \mathrm{mg} / \mathrm{l}$, respectively. The mean fosfomycin concentrations presented for different time points from 2 to $26 \mathrm{~h}$ ranged between $\sim 30$ and $66 \mathrm{mg} / \mathrm{l}$ in rabbit CSF, which roughly corresponds to drug levels achieved in CSF of humans [14]. Sterilization of CSF was achieved in all ( $\mathrm{n}=9$ out of 9 ) rabbits infected with the $S$. pneumoniae test strain with the lower MIC of $4 \mathrm{mg} / \mathrm{l}$. However, CSF sterilisation was not reached in 4 out of 9 rabbits infected with the less susceptible strain (fosfomycin MIC $16 \mathrm{mg} / \mathrm{l}$ ). In 2 of the 4 rabbits without CSF sterilization, the MIC of the strains had increased tenfold, while the other 2 rabbits were those with the lowest fosfomycin concentrations in CSF, that is, 39 and $40 \mathrm{mg} / \mathrm{l}$ after $26 \mathrm{~h}$ (overall mean 50.9 $\mathrm{mg} / \mathrm{l}$ after $26 \mathrm{~h}$ ).

Nau et al. [4] evaluated the activity of fosfomycin in a rabbit meningitis model using a $S$. penumoniae strain with an MIC of $4 \mathrm{mg} / \mathrm{l}$. Mean fosfomycin CSF concentrations of $\sim 18 \mathrm{mg} / \mathrm{l}$, corresponding to more than 4 times the MIC but markedly below the minimum bactericidal concentration (MBC) of $32 \mathrm{mg} / \mathrm{l}$, were reached but did not lead to a reduction of bacterial numbers.

\section{Comparison in vitro vs. in vivo and Conclusions}

Essentially, the in vitro experiments with $S$. aureus in $\mathrm{pH}$-adjusted CSF agree with observations in animal meningitis studies using $S$. pneumoniae in reporting that fosfomycin concentrations of a multiple of the MIC are necessary in CSF to obtain satisfactory bactericidal effects after $24 \mathrm{~h}$. However, such high ratios between fosfomycin levels and the MIC of the respective causative organism are not easily attainable in CSF in vivo. Therefore, it has been concluded that fosfomycin is unsuitable as a single agent in vivo for CNS infections, but can be used as a reserve antibiotic as part of combination therapies, for example, with ceftriaxone or vancomycin in order to enhance their activity [19].

The reasons for reduced activity of fosfomycin in CSF might at least partly be ascribed to low CSF concentrations of G-6-P in CSF, which is required for fosfomycin to enter the bacterial cell $[4,20]$. On the other hand, the general disposition of rapidly developing bacterial resistance to fosfomycin during monotherapy [21, 22] might be equally present in CSF like in other media, contributing to the observed lower effect of this drug in CSF than in MHB. A third explanation for reduced activity of fosfomycin in CSF might be that lower bacterial growth rates in CSF due to the lack of nutrients compared to broth could hamper the activity of this antibiotic known to act by interfering with bacterial murein synthesis.

In view of the differing results observed in CSF both in vitro as well as in vivo in meningitis, it can be concluded that fosfomycin microbiological experiments in broth might be misleading. In contrast, experiments in $\mathrm{pH}$-adjusted CSF in vitro seem to parallel in vivo findings in CSF infections.

\section{Rifampicin}

Mechanism of Action

Rifampicin is a lipophilic compound that specifically inhibits DNA-dependent RNA polymerase in bacterial cells, preventing transcription of bacterial DNA to RNA [23]. Killing of rifampicin was reported to be time-, growth- or exposure-dependent, depending on the target organism and the compartment of interest $[24,25]$. It easily distributes into the CNS due to its lipophilic nature.

In vitro

An in vitro study compared the activity of rifampicin against $S$. aureus in CSF with and without $\mathrm{pH}$ adjustment, and in MHB as a reference medium [13]. In non-pH-adjusted CSF, rifampicin had no bactericidal activity even at the highest concentration of $8 \times \mathrm{MIC}$, confirming the futility of microbiological experiments with CSF performed without $\mathrm{pH}$ adjustment. In $\mathrm{pH}$-adjusted $\mathrm{CSF}$, rifampicin was bactericidal at a slightly higher concentration $(2 \times$ MIC) than in MHB $(1 \times$ MIC) [13]. However, if killing occurred, it was faster in $\mathrm{pH}$-adjusted CSF than in MHB.

In vivo

Ribes et al. [26] investigated the efficacy of rifampicin against cephalosporin-resistant $S$. pneumoniae in vivo in a rabbit meningitis model with once daily rifampicin in- 
jections at a dosage of $15 \mathrm{mg} / \mathrm{kg}$ and performed a concomitant static in vitro time-kill study in an unspecified medium. For unclear reasons, different time points $(6 \mathrm{~h}$ in vitro and $24 \mathrm{~h}$ in vivo) were used for the quantification of antibacterial effects, perturbing the comparability of the data. While in vitro, only a bacteriostatic effect was described at $\geq 4 \times$ MIC, and while in vivo, there was a bactericidal effect with a mean \pm SD reduction of bacterial colony counts per $\mathrm{ml}(\Delta \log \mathrm{CFU} / \mathrm{ml})$ of $-4.25 \pm 0.91$. In the in vivo setting, the rifampicin concentrations in rabbit CSF always corresponded to a multiple of the MIC, ranging from $\sim 13 \times$ MIC (peak) to $~ 3.7 \times$ MIC (trough). Rifampicin alone was more active than vancomycin.

Nau et al. [25] found that in a model of rabbit meningitis caused by a penicillin-susceptible $S$. pneumoniae in vivo, rifampicin (MIC $0.03 \mu \mathrm{g} / \mathrm{ml}$ ) alone was significantly less bactericidal than ceftriaxone or ofloxacin. Interestingly, at maximum concentrations of rifampicin higher than $\sim 200 \times$ MIC in CSF, the killing rates decreased from $0.4 \Delta \log _{10} \mathrm{CFU} / \mathrm{ml} \times \mathrm{h}$ to $0.17 \Delta \log _{10} \mathrm{CFU} / \mathrm{ml} \times \mathrm{h}$. This phenomenon did not occur in vitro in Todd-Hewitt broth [25], and could not be explained. It was therefore attributed to the Eagle effect [27].

Comparison in vitro vs. in vivo and Conclusions

In vitro, killing of $S$. aureus by rifampicin was faster in (pH-adjusted) CSF than in broth, while a slightly higher concentration $(\geq 2 \times$ MIC) was required in CSF compared to broth $(1 \times \mathrm{MIC})$ to achieve bactericidal effects. This is in agreement with the in vivo study by Ribes et al. [26], where rifampicin achieved excellent bactericidal effects in CSF at concentrations of $\geq 3.7 \times$ MIC, whereas in broth, only bacteriostatic effects were observed. The in vivo reduction of $-4.25 \Delta \log _{10} \mathrm{CFU} / \mathrm{ml}$ observed with S. pneumoniae at the mentioned concentration range of approximately 3-13 times the MIC at $24 \mathrm{~h}$ was similar to the in vitro reduction of -3 to $-4 \Delta \log _{10} \mathrm{CFU} / \mathrm{ml}$ in CSF at $24 \mathrm{~h}$ with $S$. aureus at rifampicin concentrations ranging from 2 to 8 times the MIC [13]. Whether the finding of decreased killing rates in CSF at rifampicin concentrations higher than 200 times the MIC, as observed in vivo by Nau et al. [25], could have been reproduced in CSF in vitro is unclear because such high concentrations were not tested. Nevertheless, in summary, the data indicate that at concentrations of at least $\geq 2-4 \times$ MIC good bactericidal effects can be expected in CSF. Though it has been concluded that rifampicin is eligible for treatment of CSF infections it should be mentioned that generally rifampicin should not be administered as monotherapy in order to avoid development of resistance.

Antibiotics in CSF
Thus, there were no discrepancies between microbiological tests in $\mathrm{pH}$-adjusted CSF and in vivo results, although the comparability is limited due to the different drug concentrations achieved. In vitro tests in broth might underestimate the velocity of killing in CSF at concentrations above the MIC, whereas CSF might be a more relevant medium. When planning microbiologic experiments in vitro in CSF, the full antibiotic concentration range achieved in CSF in vivo should be tested to allow the detection of potential paradoxical microbiological phenomena like the Eagle effect.

\section{Cefepime}

Mechanism of Action

Cefepime is a 4 th-generation cephalosporin that acts, as all beta-lactams, by the inhibition of the bacterial cell wall synthesis. Its activity is growth- and time-dependent [28].

\section{In vitro}

Time-kill curves with $S$. aureus were performed in vitro in $\mathrm{pH}$-adjusted CSF, in non-pH-adjusted CSF, and in MHB [13]. In non-pH-adjusted CSF, the activity of cefepime at different concentrations was very low, and this setting was considered not relevant. After $24 \mathrm{~h}$ in $\mathrm{pH}$ adjusted CSF, killing occurred at lower concentrations $(0.5 \times$ MIC $)$ than in MHB $(2 \times$ MIC $)$. However, if killing occurred, it was slower and less pronounced in CSF than in MHB.

In vivo

Cottagnoud et al. [29] induced experimental rabbit meningitis with a penicillin- and quinolone-resistant pneumococcal strain with a cefepime MIC of $0.5 \mathrm{mg} / \mathrm{l}$. In this setting, concentrations of $\sim 10-20 \times$ MIC were reached rapidly, resulting in good bactericidal effects. Interestingly, in an accompanying in vitro experiment in $\mathrm{C}+\mathrm{Y}$ medium at a cefepime concentration of $10 \times \mathrm{MIC}$, the velocity of killing was slightly slower than in CSF in vivo [29], unlike in the in vitro study mentioned earlier [13], where killing was slower in CSF than in MHB. Differences in $\mathrm{C}+\mathrm{Y}$ medium and MHB, bacterial species, and between CSF in vitro and the in vivo situation may have contributed to this discrepancy. Täuber et al. [3] used cefepime (BMY 28142) in experimental meningitis against a susceptible $S$. pneumoniae strain. Bactericidal rates were comparable to those of other betalactams also investigated, cefpimizole and cefotaxime, and were maximized in vivo at CSF concentrations in excess of 10 times the MIC. However, because 'mean' antibiotic concentra- 
tions summarizing different time points are presented, it is not clear if concentrations were permanently at inhibitory levels or partly also at subinhibitory levels.

Gerber et al. [30] reported a mean $\Delta \log _{10} \mathrm{CFU} / \mathrm{ml} \times \mathrm{h}$ of -0.6 achieved by cefepime in a rabbit meningitis model. Two hours after the first administration, the mean CSF concentration remained between 10 and $16 \mathrm{mg} / \mathrm{l}$, which was far above the MIC of $0.5 \mathrm{mg} / \mathrm{l}$ of the tested $S$. pneumoniae strain. In this in vivo model, killing of cefepime was significantly better than the killing of ceftriaxone or vancomycin.

Comparison in vitro vs. in vivo and Conclusions

The slower cefepime killing rates observed in CSF vs. broth in vitro while killing occurred at lower cefepime concentrations in CSF compared to the artificial medium. Interestingly, this is diametrically opposed to what was reported for rifampicin in vitro. Overall, one might conclude that cefepime achieved high concentrations and satisfactory killing rates in the described in vivo experiments, as well as in vitro (in both MHB and CSF). However, the available in vitro and in vivo experiments were conducted using different bacterial test organisms with different susceptibility to cefepime. Moreover, drug concentrations reached in CSF of infected animals in the available in vivo studies were all higher than cefepime concentrations tested in the in vitro experiments in $\mathrm{pH}$ adjusted CSF. Both factors, different test organisms and different cefepime concentrations, preclude unconfounded comparability of data between the 2 settings (in vivo vs. in vitro). In summary, based on the available data, it is not possible to state whether for cefepime microbiological tests in CSF in vitro are more representative for in vivo conditions than artificial media.

\section{Cefotaxime}

Mechanism of Action

Cefotaxime is a 3rd-generation cephalosporin that acts by inhibiting the synthesis of the bacterial cell wall. As for other beta-lactams, its efficacy is determined by the time drug levels exceed the MIC [31]. Its activity is growthand time-dependent.

\section{In vitro}

According to in vitro data reported by Zhanel et al. [32], the cefotaxime MIC against 2 Escherichia coli strains was slightly higher (within one doubling dilution step, respectively) in pure $\mathrm{MHB}$ than in $\mathrm{CSF}$ ( $\mathrm{pH} 7.8$, addition of $10 \% \mathrm{MHB}$ ). Accordingly, the postantibiotic effect (PAE) of cefotaxime against $E$. coli was markedly longer in CSF (60 min) than in MHB (10 min) both after single and multiple exposures to cefotaxime concentrations twice the MIC $[32,33]$. The $\mathrm{pH}$ of the used pooled human CSF was 7.7-7.8, which is relatively close to physiologic values, and the $\mathrm{pH}$ of the employed MHB was adjusted accordingly. As an explanation for the prolonged PAE in CSF compared to MHB, the authors assumed that some protein components in the CSF might act in combination with the drug and enhance its antimicrobial activity. However, changes in PAE of cefotaxime and other drugs did not affect bacterial killing [32].

In vivo

In a model of experimental meningitis in rabbits, the mean maximum cefotaxime concentrations in CSF reached only $5.7 \%$ of corresponding serum levels, which was relatively poor compared with other tested cephalosporins. This is quite surprising since the CSF penetration of 3rd-generation cephalosporins is commonly assumed to be very good. Importantly, investigators observed significantly improved killing rates against the causative $S$. pneumoniae strain (cefotaxime MBC 0.03 $\mathrm{mg} / \mathrm{l}$ ) when CSF drug concentrations exceeded the MBC at least tenfold compared to concentrations below this threshold [3]. This was substantially confirmed by data from another rabbit meningitis model involving $2 \mathrm{~S}$. pneumoniae strains with lower susceptibility to cefotaxime (MIC $1 \mathrm{mg} / \mathrm{l}$, respectively). At comparable CSF concentrations (and presumed ratios between CSF levels and $\mathrm{MBC}$ markedly below 10), cefotaxime killing rates were in good agreement with killing rates in the lower range of those observed in the previously cited study [34].

Kim [35] reported a discrepancy between the excellent activity of cefotaxime against $E$. coli in vitro in MHB, vs. only limited killing of $E$. coli in vivo in experimental meningitis compared to other antibiotics with lower in vitro activity.

In another study, the activity of human CSF containing cefotaxime (obtained from children with meningitis who received high dose cefotaxime) was tested in vitro against cephalosporin-resistant $S$. pneumoniae strains [36]. In this approach, $10 \mu \mathrm{l}$ of inoculum (MHB with bovine serum containing cephalosporin-resistant strains) were added to $140 \mu \mathrm{l}$ of human CSF containing cefotaxime. The $\mathrm{pH}$ of this medium was not measured. Cefotaxime alone achieved the killing of some of the resistant strains, but was not reliable in all strains. The influence of adding $6.67 \%$ broth and not adjusting $\mathrm{pH}$ can hardly be estimated. 
Comparison in vitro vs. in vivo and Conclusions

The in vitro tests described a favourable effect of CSF on PAE of cefotaxime compared to broth. However, this did neither affect bacterial killing, which was comparable between the 2 media in the same set of in vitro experiments, nor was a supposed activity-enhancing influence of CSF on cefotaxime observed in the evaluated in vivo models. In addition, existing data stem from experiments conducted with different test organisms and in different growth media. Overall, it is therefore not possible to assess whether the activity of cefotaxime in vivo in CSF can be predicted better with experiments in CSF in vitro than with experiments in broth. Importantly, low CSF concentrations of cefotaxime as observed in one of the in vivo studies might suggest caution in the use of this drug for therapy of CNS infections.

\section{Ceftriaxone}

Mechanism of Action

Ceftriaxone is a 3rd-generation cephalosporin acting through the inhibition of bacterial cell wall synthesis. It is highly protein bound and has an exceptionally long serum half-life $[37,38]$. A PAE is described against various Gram-positive and Gram-negative organisms [39-41].

\section{In vitro}

Decazes et al. [42] found MIC and MBC of ceftriaxone against a clinical $E$. coli isolate to be identical in pooled rabbit CSF and MHB $(0.06 \mu \mathrm{g} / \mathrm{ml}$, respectively). In timekill studies against the same isolate, drug concentrations of up to 3 times the MBC showed improved bactericidal activity in MHB compared to CSF (colony count reduction after $7 \mathrm{~h}=-2.5 \log \mathrm{CFU} / \mathrm{ml}$ in MHB vs. $-1.5 \log \mathrm{CFU} /$ $\mathrm{ml}$ in $\mathrm{CSF}$ ), whereas only ceftriaxone concentrations greater than 10 times the $\mathrm{MBC}$ produced maximal bactericidal effect, irrespective of the employed medium. $\mathrm{pH}$ adjustment was not mentioned in the manuscript.

\section{In vivo}

Within the same manuscript, antimicrobial killing activity of ceftriaxone against the same E. coli strain was assessed in an experimental meningitis model in rabbits in vivo. Here, mean killing rates of ceftriaxone at concentrations up to 10 times the MBC amounted to $-0.7 \log \mathrm{CFU} /$ $\mathrm{ml}$ per hour. Above this threshold, that is, at ceftriaxone levels exceeding concentrations greater than 10 times the $\mathrm{MBC}$, the mean colony reduction was $-1.5 \log \mathrm{CFU} / \mathrm{ml}$ per hour [42].

Gerber et al. [43] investigated the activity of ceftriaxone against a clinical penicillin-sensitive $S$. pneumoniae type 3 isolate (MIC and MBC for ceftriaxone 0.03 and $0.06 \mu \mathrm{g} / \mathrm{ml}$, respectively) in a rabbit meningitis model. At reported CSF concentrations of approximately $5 \mu \mathrm{g} / \mathrm{ml}$, ceftriaxone produced killing rates of $-0.64 \pm 0.16 \mathrm{CFU} /$ $\mathrm{ml}$ per hour.

In another experimental pneumococcal meningitis model (MIC of the test organism $0.023 \mu \mathrm{g} / \mathrm{ml}$ ), ceftriaxone led to the reduction of bacterial counts in CSF of infected rabbits of approximately $-0.7 \log \mathrm{CFU} / \mathrm{ml}$ per hour [44]. Ceftriaxone was administered at a single subcutaneous dose of $100 \mathrm{mg} / \mathrm{kg}$ and concentrations attained in CSF were not measured.

Analogous to previous observations, Nau et al. [5] reported optimized antimicrobial activity of ceftriaxone in CSF of rabbits infected with a penicillin-susceptible $S$. pneumoniae type 3 strain at CSF drug concentrations exceeding the $\mathrm{MBC}$ by several fold. Bactericidal rates were $-0.57 \log \mathrm{CFU} / \mathrm{ml}$ per hour at a mean ceftriaxone concentration in CSF/MBC ratio of 12.3 and $-0.80 \log \mathrm{CFU} / \mathrm{ml}$ per hour at a mean ceftriaxone concentration in $\mathrm{CSF} /$ $\mathrm{MBC}$ ratio of 83.3 , respectively.

\section{Comparison in vitro vs. in vivo and Conclusions}

Evidence from the 3 cited pneumococcal meningitis models seems to confirm the need for CSF concentrations several fold superior to the MIC/MBC in order to guarantee optimized bactericidal activity, even though CSF concentrations were not measured in one study. Data on susceptibility of $S$. pneumoniae to ceftriaxone in CSF in vitro are to date not available.

In the studies by Decazes et al. [42], bactericidal activity of ceftriaxone in time-kill curves in CSF in vitro was in good agreement with in vivo efficacy observed in E. coli meningitis. As limitations, presented outcome parameters (killing rates vs. CFU reduction at a single time point) were not exactly superimposable between in vitro and in vivo experiments, and $\mathrm{pH}$ adjustment was apparently not performed. Still, these findings might indicate that in vitro tests in CSF can contribute to predict ceftriaxone's ability to treat E. coli meningitis. As pointed out by the authors, time-kill curves might be superior to MIC/MBC determination in this regard.

\section{Ciprofloxacin}

\section{Mechanism of Action}

Ciprofloxacin is a broad-spectrum fluoroquinolone antibiotic. Its activity is concentration dependent and relies on the inhibition of the bacterial DNA gyrase [45]. A concentration-dependent PAE is described for a number of both Gram-positive and Gram-negative organisms [46-48]. 
In vitro

Zhanel et al. [32] measured MIC and PAE of ciprofloxacin against $2 \mathrm{E}$. coli strains in pooled human CSF (90\% CSF, 10\% MHB) and cation-adjusted MHB. The $\mathrm{pH}$ of CSF was approximately 7.8, which is relatively close to physiologic values, and the $\mathrm{pH}$ of $\mathrm{MHB}$ was adjusted accordingly. While MIC values in both media were within one doubling dilution step, ciprofloxacin showed a markedly longer PAE in CSF (range 130-160 min) compared to $\mathrm{MHB}$ (70-110 $\mathrm{min})$. In another study, after multiple ciprofloxacin doses, MIC values reportedly increased both in MHB and CSF. Interestingly, after repeated exposure to ciprofloxacin, PAE was observed to decrease in MHB but remained stable in CSF [33]. As a limitation, CSF solutions seem to have been incubated in ambient air and not in a $\mathrm{CO}_{2}$-containing atmosphere, which might have led to an increase in $\mathrm{pH}$ and might in turn have altered bacterial growth and/or antibacterial activity of ciprofloxacin.

\section{In vivo}

Nau et al. [5] tested CSF penetration and bactericidal activity of 2 different intravenous doses of ciprofloxacin in a rabbit model of pneumococcal meningitis. At the lower dose of $10 \mathrm{mg} / \mathrm{kg} / \mathrm{h}$, ciprofloxacin concentrations in CSF over a 7-hour observation period ranged from 0.5 to $1.6 \mu \mathrm{g} / \mathrm{ml}$, corresponding to $0.5-1.6$ times the ciprofloxacin MIC for the causative S. pneumoniae strain. No inhibition of bacterial growth was observed. The higher ciprofloxacin dose $(40 \mathrm{mg} / \mathrm{kg} / \mathrm{h})$ led to CSF concentrations ranging from 3.5 to $10.7 \mu \mathrm{g} / \mathrm{ml}$, corresponding to 3.5-10.7 times the MIC. Here, ciprofloxacin showed only weak bactericidal activity, measured as a reduction in bacterial counts of $-0.24 \log \mathrm{CFU} / \mathrm{ml} / \mathrm{h}$. However, as noted by the authors, the suitability of older quinolones such as ciprofloxacin for the treatment of Gram-positive CNS infections had already been questioned [49].

Comparison in vitro vs. in vivo and Conclusions

The 2 studies listed above are difficult to compare due to differences in experimental setting, measured outcome parameters and employed bacterial strains. On the one hand, the observed prolonged PAE of ciprofloxacin in CSF might well be a favourable feature of the drug in the treatment of CSF infections. Nevertheless, as long as it does not correlate with an improved antibacterial activity in vivo, the clinical relevance of this finding must be considered limited. To date, the available evidence does not allow to state whether antibacterial efficacy in CSF of ciprofloxacin in vivo can be predicted by in vitro tests.

\section{Gentamicin}

Mechanism of Action

Gentamicin is a broad-spectrum aminoglycoside antibiotic that acts by the inhibition of bacterial protein synthesis through irreversible binding to the $30 \mathrm{~S}$ ribosome subunit. It displays rapid bactericidal, concentration-dependent activity against a wide range of Gram-negative bacteria and has a PAE against anaerobic and facultative Gram-negative rods [50].

In vitro and in vivo

Data available for gentamicin comprise several sets of both in vitro and in vivo experiments performed and published at once by the respective research groups, and are therefore presented in one comprehensive section.

In analogy to in vitro experiments with ciprofloxacin, Zhanel et al. [32] observed a prolonged PAE in CSF compared to $\mathrm{MHB}$ also for gentamicin. Time without regrowth of 2 E. coli strains after removal of gentamicin was markedly longer in CSF (range 180-250 min) than in MHB (10-130 min). Similarly to what was reported for ciprofloxacin, gentamicin MIC values in CSF were within one doubling dilution step from those in MHB. After multiple gentamicin doses, bacterial killing of gentamicin declined both in MHB and CSF, while its PAE was observed to decrease in MHB but increase in CSF. As the underlying cause for this, the authors hypothesized an interaction between a still unknown component of CSF and gentamicin [33]. However, although a prolonged PAE in CSF might indicate improved activity of gentamicin when used to treat CNS infections, these experiments were affected by the flaws mentioned earlier for ciprofloxacin (most notably, no $\mathrm{pH}$ adjustment was described) and might therefore not be entirely reliable.

Cottagnoud et al. [51] performed concomitant in vitro and in vivo experiments with gentamicin against a penicillin-resistant S. pneumoniae strain (gentamicin MIC $4 \mathrm{mg} / \mathrm{l})$. In vitro, killing rates of gentamicin against the test organism did not exceed $-0.1 \log \mathrm{CFU} / \mathrm{ml}$ after $8 \mathrm{~h}$ of incubation in $\mathrm{C}+\mathrm{Y}$ medium. In an experimental meningitis model in rabbits, the penetration of gentamicin into CSF was low and drug levels were observed to fall below the MIC already after approximately $4 \mathrm{~h}$. However, at almost identical drug concentrations and using the same test strain, antimicrobial activity of gentamicin was slightly better in CSF in vivo than in $\mathrm{C}+\mathrm{Y}$ medium in vitro $(\Delta \log \mathrm{CFU} / \mathrm{ml}$ at $8 \mathrm{~h}=-1.24)$.

In contrast to these findings, which seem to point towards an enhanced antibacterial activity of gentamicin in association with CSF, Scheld et al. [52] conducted in vitro 
experiments where gentamicin was observed to be less active in CSF than in broth (heart infusion broth): gentamicin was tested against a clinical bloodstream isolate of E. coli at concentrations of $2,4,8$, and $16 \mathrm{mg} / \mathrm{l}$, corresponding to $1,2,4$, and 8 times the MIC (and MBC), respectively. At $2 \mathrm{mg} / \mathrm{l}$, gentamicin achieved a killing of -3.2 $\log \mathrm{CFU} / \mathrm{ml}$ after $8 \mathrm{~h}$ in both media. In CSF, killing was not improved at gentamicin concentrations higher than $2 \mathrm{mg} / \mathrm{l}$, while heart infusion broth spiked with higher gentamicin concentrations was sterile by $2-8 \mathrm{~h}$. $\mathrm{pH}$ changes in CSF and broth were not specifically measured over time but incubation with $5 \% \mathrm{CO}_{2}$ indicates presumably physiological values.

In an experimental rabbit meningitis model using the same $E$. coli strain, a mean gentamicin CSF concentration of approximately $3 \mathrm{mg} / \mathrm{l}$ (equivalent to approximately 1.5 times the MIC) resulted in a $\Delta \log \mathrm{CFU} / \mathrm{ml}$ of +0.35 after $8 \mathrm{~h}$, whereas only higher CSF concentrations achieved modest killing. The highest killing rate, corresponding to an 8-hour $\Delta \log C F U / m l$ of -2 , was observed at a CSF concentration of $14.2 \mathrm{mg} / \mathrm{l}$, corresponding to approximately 7 times the MIC [52].

In a different $E$. coli rabbit meningitis model investigated by the same researchers, a mean gentamicin CSF concentration of $2.6 \mathrm{mg} / \mathrm{l}$ (corresponding to 2.6 times the $\mathrm{MBC}$ for the test organism) was shown to achieve a reduction of bacterial colonies of $-3.4 \mathrm{CFU} / \mathrm{ml}$ over $8 \mathrm{~h}$ [10].

Comparison in vitro vs. in vivo and Conclusions

Taken together, the information evincible from available studies is somewhat conflicting. On the one hand, the CSF-associated prolongation of gentamicin PAE reported by Zhanel et al. [32] and Karlowsky et al. [33] points towards a favourable effect of CSF on the antibacterial efficacy of gentamicin, mediated by a yet to be identified component of serum and/or CSF. This might be seen in agreement with the slight improvement of gentamicin killing in CSF compared to broth observed by Cottagnoud et al. [51]. Good antibacterial activity of gentamicin in CSF was also shown by Scheld et al. [52] in one of the cited meningitis models.

These results are, however, in contrast to data presented by Cottagnoud et al. [51] and Scheld et al. [52] which describe rather low killing rates of gentamicin in CSF compared to other growth media. In any case, as for other drugs discussed in this review, divergences in test media $(\mathrm{C}+\mathrm{Y}$ medium, heart infusion broth), settings (e.g. strains) and insufficiently reported details on killing rates, incubation measures and $\mathrm{pH}$ changes preclude unambig- uous statements on the ability of in vitro time-kill studies in CSF to predict gentamicin's activity in the treatment of bacterial meningitis.

\section{Vancomycin}

Mechanism of Action

Vancomycin, a glycopeptide antibiotic, is bactericidal against most gram-positive bacteria through the inhibition of peptidoglycan synthesis [53].

\section{In vitro}

Vancomycin was tested in time-kill studies in CSF in vitro against S. aureus ATCC 25923, S. epidermidis ATCC 12228 , and a clinical isolate of methicillin-resistant $S$. epidermidis [54]. The MIC for vancomycin was $2 \mu \mathrm{g} / \mathrm{ml}$ for all strains. Experiments were conducted in samples of human CSF collected from patients undergoing lumbar puncture for various indications. Importantly, CSF samples from patients undergoing antimicrobial treatment, samples with bacterial contamination as well as samples showing antimicrobial activity were excluded. Killing kinetics of vancomycin was assessed at 2, 5, 10, 100 and 300 $\mu \mathrm{g} / \mathrm{ml}$, respectively. Reduction of viable counts by $3 \mathrm{log}$ $\mathrm{CFU} / \mathrm{ml}$ could be achieved only after $24-48 \mathrm{~h}$, and only at vancomycin concentrations equal to or higher than $5 \mu \mathrm{g} / \mathrm{ml}$. Vancomycin concentrations of 10,100 and $300 \mu \mathrm{g} / \mathrm{ml}$ did not significantly improve killing compared to $5 \mu \mathrm{g} / \mathrm{ml}$. No $\mathrm{pH}$ adjustment measures were reported.

In vivo

The activity of vancomycin in human CSF in vivo was determined in a pediatric patient with a ventriculoperitoneal shunt infection caused by the same methicillin-resistant S. epidermidis isolate mentioned earlier [54]. As observed in CSF in vitro, the colony count reduction was slow. Starting from a bacterial burden of $6 \times 10^{3}$ to $2 \times 10^{4}$ $\mathrm{CFU} / \mathrm{ml}$ before initiation of therapy, sterile CSF (compatible with a reduction in bacterial counts of $>3 \log$ CFU/ $\mathrm{ml}$ ) could be achieved only after 4 days. Vancomycin concentrations in CSF samples drawn at days 2 and 3 were 4 and $6 \mu \mathrm{g} / \mathrm{ml}$, respectively.

\section{Comparison in vitro vs. in vivo and Conclusions}

Given their paucity, available data must be interpreted very prudently, and making an inference of definite conclusions is not possible. In the clinical case report, in vivo activity of vancomycin was in good agreement with data from in vitro experiments in CSF. However, although the same test organism was used, CFU counts and vancomycin concentrations in CSF in vivo were measured only 
sporadically during the course of treatment. This prevented the accurate calculation of killing rates and led to an inhomogeneous data presentation between in vitro and in vivo studies. Also, as for other agents, it seems that no $\mathrm{pH}$ adjustment was performed in vitro. Furthermore, inclusion of additional time-kill studies in conventional MHB in the in vitro experiments would have been recommendable. Since only CSF was used, it is not possible to state whether in vitro tests in CSF are superior to experiments in other growth media in predicting vancomycin's activity in vivo.

\section{Summary, Limitations, Outlook and Conclusions}

To our knowledge, this is the first review article systematically dealing with the question as to what extent the antimicrobial activity of antibiotics is influenced by CSF and whether in vitro experiments with antimicrobial drugs meant to treat CNS infections can predict their in vivo efficacy. This approach is of importance for clinical practice, since it might contribute to understand why certain drugs succeed in treating, or fail to treat, potentially life-threatening infections such as meningitis. In addition, this compilation of the available evidence on the topic helped to identify a series of methodological limitations of both in vitro and in vivo experiments conducted so far. Precautions and recommendations derived from these observations might contribute to optimize future investigations on the antimicrobial activity in CSF of drugs meant to treat CNS infections, and might be of particular interest for researchers involved in preclinical drug development.

In this article, we summarized available in vitro data generated in CSF and broth and matched them with comparable in vivo studies. A definitive answer to the question whether in vitro studies in CSF can generally predict the ability of a drug to treat bacterial meningitis in vivo cannot be provided to date. Although there are indications that this principle is applicable and helpful for some antibiotics, there are several limitations. The availability of CSF as a microbiological medium is restricted, and data on bacterial killing kinetics of antimicrobial drugs in CSF in vitro are extremely rare. Moreover, a large portion of the results and comparisons compiled here stems from differently designed experiments. This has resulted in implications on the comparability of the investigated outcomes and is therefore profoundly linked to the conclusions that can be drawn from them.

Each of the following paragraphs is dedicated to one of the major limitations of the studies discussed in this re- view. The recommendations that can be derived from them are summarized in italic characters at the end of each paragraph.

One crucial aspect, repeatedly mentioned in this article, is constituted by $\mathrm{pH}$ adjustment measures in in vitro experiments involving CSF. $\mathrm{pH}$ alterations in CSF can markedly affect the growth and killing of bacterial test organisms in vitro, leading to wrong estimates of the activity of the investigated drug. Not all investigators determined $\mathrm{pH}$ values or reported $\mathrm{pH}$ adjustment measures in CSF in their in vitro experiments, restricting both the reliability of their own results as well as their comparability to data from other studies. The fact that the in vitro studies conducted in $\mathrm{pH}$ adjusted CSF (i.e. for fosfomycin and rifampicin, respectively) were those with the best correlation to in vivo efficacy of the respective substances might serve as additional proof of the relevance of $\mathrm{pH}$ adjustment in microbiological experiments with CSF.

Care should be taken to perform $\mathrm{pH}$ adjustment of all employed growth media. In this regard, incubation of CSF with $5 \% \mathrm{CO}_{2}$ is an easily applicable and reliable method.

Moreover, the available studies for one single antimicrobial compound were often conducted using different bacterial strains for in vitro and in vivo experiments, respectively. Here, establishing direct correlations between the 2 settings is problematic due to diverging susceptibility patterns and other differing properties of the isolates. Similarly, clear comparisons between in vitro and in vivo studies were sometimes not feasible due to different outcome parameters (PAE vs. bacterial kill rates), divergent patterns of data presentation (kill rates over a defined time period vs. punctual colony count reduction at a single time point compared to baseline) or discrepancies in time points chosen for efficacy evaluation. These reasons impede univocal statements on predictability of in vivo efficacy based on CSF experiments for the antibiotics cefepime, cefotaxime and ciprofloxacin, even though relatively sound in vitro results are available.

Whenever the antimicrobial activity of an antibiotic agent designed for treatment of CNS infections is assessed in CSF both in vitro and in vivo, care should be taken to ensure comparability of bacterial test organisms and investigated outcome parameters between different experimental settings and with existing evidence.

Finally, similar considerations also apply to different microbiological growth media. MHB, the probably bestcharacterized medium used for bacterial susceptibility testing, is known to favour bacterial growth due to its high content of nutrients such as glucose and selected electrolytes. The variety of growth media employed in the in vi- 
tro experiments included in this review (MHB, C + Y medium, heart infusion broth, Schaedler medium) are likely to go along with altered bacterial growth patterns and accordingly account for changes in killing rates of antimicrobial drugs, in particular of those known to act in a growth-dependent manner.

In microbiological in vitro experiments with CSF, we suggest that MHB is employed as a control medium, if applicable. If not applicable, investigators should be aware of control growth media used in existing studies and accordingly choose a growth medium with identical or similar composition.

One aspect that was highlighted and agreed upon by a majority of studies enlisted here is that drug concentrations in CSF need to be a multiple of the MIC/MBC of the respective target organism for optimal antibacterial killing. In this regard, most studies indicate a concentration threshold of 10 times the MIC/MBC, which might well correspond to very high drug levels. However, in some cases the killing rates observed at very high drug concentrations in CSF in vivo could not be correlated to in vitro kinetics simply because such high concentrations were not tested in vitro.

In vitro experiments with antimicrobial drugs in human CSF should include a range of concentrations representative of drug levels reached in human CSF during CNS infections. In this context, in vitro simulations of the pharmacokinetic profile of the drug of interest in CSF in vivo might be of particular value. In general, in order to improve comparability, care should be taken to choose identical or similar concentration levels in different experimental settings. Again, awareness of investigators about existing data is crucial.

An additional aspect that has to be kept in mind is constituted by inflammation-induced changes in CSF composition. Under inflammatory conditions, CSF composition is known to vary considerably due to, for instance, increase in protein content, decrease in glucose levels, blood or pus contamination. The potential impact of these changes on pharmacokinetic and/or pharmacodynamic properties of antimicrobial drugs is heterogenous and can hardly be quantified.

The potential impact of inflammation-associated changes in CSF composition on antimicrobial activity of CNS-active drugs should be considered in the design, performance and interpretation of in vivo models of CNS infections.

In conclusion, studies performed under optimal experimental conditions seem to indicate that in vitro experiments in human CSF are superior to those in artificial media in predicting in vivo efficacy of selected antimicrobial drugs meant to treat CNS infections. Nevertheless, methodological flaws and restricted comparability of the majority of the available in vitro and in vivo studies preclude scientifically sound statements in this regard. Based on the limitations of the collected studies, this review tried to compile methodological precautions that might contribute to improve the comparability of future investigations on antimicrobial activity of antibiotics in CSF.

\section{References}

1 Friedland IR, et al: Evaluation of antimicrobial regimens for treatment of experimental penicillin- and cephalosporin-resistant pneumococcal meningitis. Antimicrob Agents Chemother 1993;37:1630-1636.

2 McCracken GH Jr, Sakata Y: Antimicrobial therapy of experimental meningitis caused by Streptococcus pneumoniae strains with different susceptibilities to penicillin. Antimicrob Agents Chemother 1985;27:141-145.

3 Täuber MG, et al: New cephalosporins cefotaxime, cefpimizole, BMY 28142, and HR 810 in experimental pneumococcal meningitis in rabbits. Antimicrob Agents Chemother 1985; 27:340-342.

4 Nau R, et al: Activity of fosfomycin in a rabbit model of experimental pneumococcal meningitis. J Antimicrob Chemother 1995;36:9971004.

5 Nau R, et al: Quinolone antibiotics in therapy of experimental pneumococcal meningitis in rabbits. Antimicrob Agents Chemother 1995; 39:593-597.

6 Lutsar I, McCracken GH Jr, Friedland IR: Antibiotic pharmacodynamics in cerebrospinal fluid. Clin Infect Dis 1998;27:1117-1127; quiz 1128-1129.

7 Segal MB: Extracellular and cerebrospinal fluids. J Inherit Metab Dis 1993;16:617-638.

8 Kandel ER, Schwartz JH, Jessell TM: Principles of Neural Science, ed 4. McGraw-Hill, 2000.

9 Hasbun R: Cerebrospinal fluid in central nervous system infections; in Scheld WM, Whitley RJ, Marra CM (eds): Infections of the Central Nervous System. Philadelphia, Wolters Kluwer Health, 2014.

10 Scheld WM, et al: Cerebrospinal fluid outflow resistance in rabbits with experimental meningitis. Alterations with penicillin and methylprednisolone. J Clin Invest 1980;66:243253.
11 Nau R, Sörgel F, Prange HW: Pharmacokinetic optimisation of the treatment of bacterial central nervous system infections. Clin Pharmacokinet 1998;35:223-246.

12 Cunniffe JG, Whitby-Strevens S, Wilcox MH: Effect of $\mathrm{pH}$ changes in cerebrospinal fluid specimens on bacterial survival and antigen test results. J Clin Pathol 1996;49:249-253.

13 Sauermann R, et al: Antimicrobial activity of cefepime and rifampicin in cerebrospinal fluid in vitro. J Antimicrob Chemother 2008;62: 1057-1060.

14 Pfausler B, et al: Concentrations of fosfomycin in the cerebrospinal fluid of neurointensive care patients with ventriculostomy-associated ventriculitis. J Antimicrob Chemother 2004;53:848-852.

15 Kühnen E, Pfeifer G, Frenkel C: Penetration of fosfomycin into cerebrospinal fluid across non-inflamed and inflamed meninges. Infection 1987; 15:422-424. 
16 Brunner M, et al: Penetration of fosfomycin into the parenchyma of human brain: a case study in three patients. $\mathrm{Br} \mathrm{J}$ Clin Pharmacol 2002;54:548-550.

17 Pfeifer G, Frenkel C, Entzian W: Pharmacokinetic aspects of cerebrospinal fluid penetration of fosfomycin. Int J Clin Pharmacol Res 1985;5:171-174.

18 Sauermann R, et al: Cerebrospinal fluid impairs antimicrobial activity of fosfomycin in vitro. J Antimicrob Chemother 2009;64:821-823.

19 Ribes S, et al: Evaluation of fosfomycin alone and in combination with ceftriaxone or vancomycin in an experimental model of meningitis caused by two strains of cephalosporinresistant Streptococcus pneumoniae. J Antimicrob Chemother 2006;57:931-936.

20 Dette GA, et al: Comparative study of fosfomycin activity in Mueller-Hinton media and in tissues. J Antimicrob Chemother 1983;11: 517-524.

21 Ellington MJ, et al: Mutators among CTX-M beta-lactamase-producing Escherichia coli and risk for the emergence of fosfomycin resistance. J Antimicrob Chemother 2006;58 848-852.

22 Souli M, et al: In vitro interactions of antimicrobial combinations with fosfomycin against KPC-2-producing Klebsiella pneumoniae and protection of resistance development. Antimicrob Agents Chemother 2011;55:2395-2397.

23 Wehrli W: Rifampin: mechanisms of action and resistance. Rev Infect Dis 1983;5(suppl 3):S407-S411.

24 van Ingen J, et al: Why do we use $600 \mathrm{mg}$ of rifampicin in tuberculosis treatment? Clin Infect Dis 2011;52:e194-e199.

25 Nau R, et al: Rifampin for therapy of experimental pneumococcal meningitis in rabbits. Antimicrob Agents Chemother 1994;38: 1186-1189.

26 Ribes S, et al: Evaluation of ceftriaxone, vancomycin and rifampicin alone and combined in an experimental model of meningitis caused by highly cephalosporin-resistant Streptococcus pneumoniae ATCC 51916. J Antimicrob Chemother 2005;56:979-982.

27 Eagle H, Musselman AD: The rate of bactericidal action of penicillin in vitro as a function of its concentration, and its paradoxically reduced activity at high concentrations against certain organisms. J Exp Med 1948;88:99-131.

28 Ambrose PG, et al: Pharmacodynamic considerations in the treatment of moderate to severe pseudomonal infections with cefepime. J Antimicrob Chemother 2002;49: 445-453.

29 Cottagnoud $\mathrm{P}$, et al: Cefepime is efficacious against penicillin- and quinolone-resistant pneumococci in experimental meningitis. J Antimicrob Chemother 2002;49:327-330.
30 Gerber CM, et al: Evaluation of cefepime alone and in combination with vancomycin against penicillin-resistant pneumococci in the rabbit meningitis model and in vitro. J Antimicrob Chemother 2000;45:63-68.

31 Turnidge JD: Pharmacodynamic (kinetic) considerations in the treatment of moderately severe infections with cefotaxime. Diagn Microbiol Infect Dis 1995;22:57-69.

32 Zhanel GG, et al: Effect of pooled human cerebrospinal fluid on the postantibiotic effects of cefotaxime, ciprofloxacin, and gentamicin against Escherichia coli. Antimicrob Agents Chemother 1992;36:1136-1139.

33 Karlowsky JA, et al: In vitro postantibiotic effects following multiple exposures of cefotaxime, ciprofloxacin, and gentamicin against Escherichia coli in pooled human cerebrospinal fluid and Mueller-Hinton broth. Antimicrob Agents Chemother 1993;37:1154-1157.

34 Kühn F, et al: Cefotaxime acts synergistically with levofloxacin in experimental meningitis due to penicillin-resistant pneumococci and prevents selection of levofloxacin-resistant mutants in vitro. Antimicrob Agents Chemother 2003;47:2487-2491.

35 Kim KS: Comparison of cefotaxime, imipenem-cilastatin, ampicillin-gentamicin, and ampicillin-chloramphenicol in the treatment of experimental Escherichia coli bacteremia and meningitis. Antimicrob Agents Chemother 1985;28:433-436.

36 Friedland IR, Klugman KP: Cerebrospinal fluid bactericidal activity against cephalosporin-resistant Streptococcus pneumoniae in children with meningitis treated with highdosage cefotaxime. Antimicrob Agents Chemother 1997;41:1888-1891.

37 Seddon M, et al: Pharmacokinetics of Ro 139904, a broad-spectrum cephalosporin. Antimicrob Agents Chemother 1980;18:240-242.

38 Stoeckel K, et al: Effects of concentration-dependent plasma protein binding on ceftriaxone kinetics. Clin Pharmacol Ther 1981;29: 650-657.

39 Ravizzola G, et al: Ceftriaxone against gramnegative and gram-positive bacteria: bactericidal and post-antibiotic effect. Chemioterapia 1985;4:204-208.

40 Buxbaum A, Georgopoulos A: Postantibiotic effect of ceftriaxone and gentamicin alone and in combination on Klebsiella pneumoniae, Pseudomonas aeruginosa and Streptococcus viridans. Infection 1996;24:459-464.

41 Aldridge KE: Comparison of the post-antibiotic effect (PAE) induced by ceftizoxime, ceftriaxone, cefoxitin, ampicillin-sulbactam, and ticarcillin-clavulanate against selected isolates of Bacteroides fragilis and B. thetaiotaomicron. Anaerobe 2002;8:295-299.
42 Decazes JM, Ernst JD, Sande MA: Correlation of in vitro time-kill curves and kinetics of bacterial killing in cerebrospinal fluid during ceftriaxone therapy of experimental Escherichia coli meningitis. Antimicrob Agents Chemother 1983;24:463-467.

43 Gerber J, et al: Rifampin followed by ceftriaxone for experimental meningitis decreases lipoteichoic acid concentrations in cerebrospinal fluid and reduces neuronal damage in comparison to ceftriaxone alone. Antimicrob Agents Chemother 2003;47:13131317.

44 Grandgirard D, et al: Attenuation of cerebrospinal fluid inflammation by the nonbacteriolytic antibiotic daptomycin versus that by ceftriaxone in experimental pneumococcal meningitis. Antimicrob Agents Chemother 2010; 54:1323-1326.

45 Wolfson JS, Hooper DC: The fluoroquinolones: structures, mechanisms of action and resistance, and spectra of activity in vitro. Antimicrob Agents Chemother 1985;28:581586

46 Odenholt I, Löwdin E, Cars O: Postantibiotic, postantibiotic sub-MIC, and subinhibitory effects of PGE-9509924, ciprofloxacin, and levofloxacin. Antimicrob Agents Chemother 2003;47:3352-3356.

47 Kumar A, et al: Post-antibiotic effect of ceftazidime, ciprofloxacin, imipenem, piperacillin and tobramycin for Pseudomonas cepacia. J Antimicrob Chemother 1992;30: 597-602.

48 Fuursted K: Post-antibiotic effect of ciprofloxacin on Pseudomonas aeruginosa. Eur J Clin Microbiol 1987;6:271-274.

49 Scheld WM: Quinolone therapy for infections of the central nervous system. Rev Infect Dis 1989;11(suppl 5):S1194-S1202.

50 Freeman CD, et al: Once-daily dosing of aminoglycosides: review and recommendations for clinical practice. J Antimicrob Chemother 1997;39:677-686.

51 Cottagnoud P, et al: Gentamicin increases the efficacy of vancomycin against penicillin-resistant pneumococci in the rabbit meningitis model. Antimicrob Agents Chemother 2002; 46:188-190.

52 Scheld WM, Brown RS Jr, Sande MA: Comparison of netilmicin with gentamicin in the therapy of experimental Escherichia coli meningitis. Antimicrob Agents Chemother 1978;13:899-904.

53 Nagarajan R: Antibacterial activities and modes of action of vancomycin and related glycopeptides. Antimicrob Agents Chemother 1991;35:605-609.

54 Nagl M, et al: Bactericidal activity of vancomycin in cerebrospinal fluid. Antimicrob Agents Chemother 1999;43:1932-1934. 